\title{
Ecthyma gangrenosum of a single limb
}

\author{
George M. Varghese, Pushpa Eapen ${ }^{1}$, Susanne Abraham ${ }^{1}$
}

Ecthyma gangrenosum is a skin manifestation of systemic sepsis commonly caused by Pseudomonas aeruginosa in patients with neutropenia or underlying immune deficiency. Although the usual outcome is poor, early recognition and appropriate systemic antibiotic treatment can lead to successful outcome. We report a case of a previously healthy lady with no apparent immune deficiency or neutropenia who had ecthyma gangrenosum of left lower limb in which the arterial line was placed.

Keywords: Ecthyma gangrenosum, Pseudomonas aeruginosa, single limb

\begin{tabular}{|l|}
\hline Access this article online \\
\hline Website: www.ijccm.org \\
\hline DOI: 10.4103/0972-5229.84898 \\
\hline Quick Response Code: \\
\hline \\
\hline
\end{tabular}

\section{Introduction}

Ecthyma gangrenosum is a known skin manifestation of severe systemic infection commonly due to Pseudomonas aeruginosa. Most often it is seen in immunocompromised or neutropenic patients who present with skin lesions that begin as an erythematous nodule or hemorrhagic vesicle, which evolves into a necrotic ulcer with eschar. ${ }^{[1]}$ The skin lesions are usually widespread over the body and the case fatality rate is high. We report a case of ecthyma gangrenosum of left lower limb following arterial line in the left femoral artery in an individual with no apparent immune deficiency or neutropenia.

\section{Case Report}

A 23-year-old woman was admitted to Medical Intensive Care Unit with organophosphorus poisoning. The patient required mechanical ventilation and ionotropic support. Left femoral artery catheter was inserted for blood pressure monitoring. On the seventh hospital day she developed fever $\left(102^{\circ} \mathrm{F}\right)$ and

\section{From:}

Departments of Medicine and Infectious Diseases, and 'Dermatology,

Christian Medical College, Vellore, India

\section{Correspondence:}

Dr. George M. Varghese, Department of Medicine and Infectious Diseases, Christian Medical College, Vellore - 632004, India.

E-mail: georgemvarghese@ hotmail.com thachycardia. Three days later the patient was noted to have erythematous papules on the left lower limb. The white blood cell count was $16,500 / \mathrm{mm}^{3}$ (neutrophils $84 \%$, lymphocytes $12 \%$, monocytes $3 \%$, eosinophils $1 \%$ ). Two sets of blood cultures were sent and the intra-arterial catheter was removed. The skin lesions were biopsied and sent for histopathology and culture. Over the next few days the skin lesions became blackish with necrotic areas (arrow) [Figure 1]. The blood, catheter tip, and skin lesion cultures yielded Pseudomonas aeruginosa sensitive to ceftazidime. Biopsy of the skin lesion revealed acute

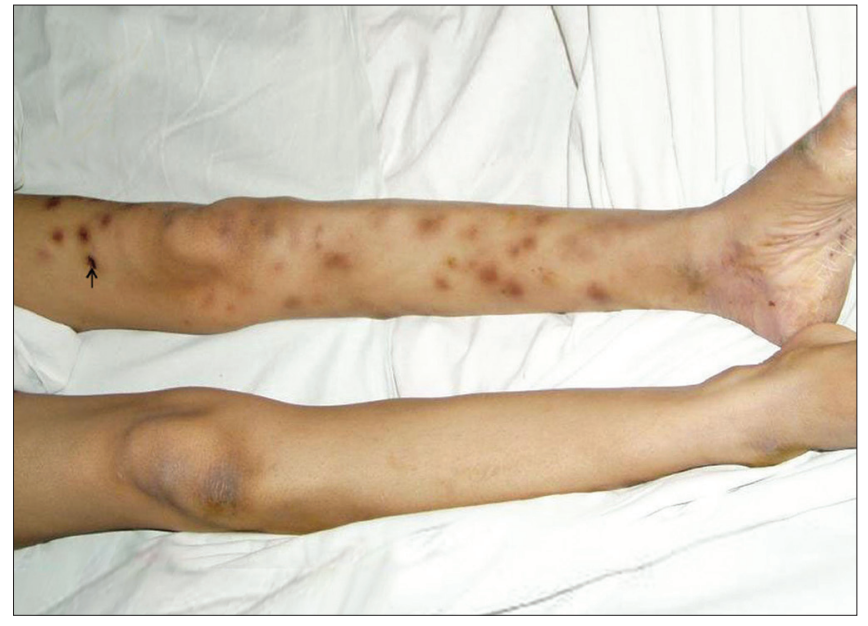

Figure I: Multiple erythematous papules and a few black necrotic lesions (arrow) on the left lower limb, suggestive of ecthyma gangrenosum 
neutrophilic infiltration pronounced in the perivascular area with necrotic area. The patient was treated with ceftazidime for 2 weeks and lesions resolved completely. Subsequently the patient had full recovery from organophosphorus poisoning and was discharged home.

\section{Discussion}

Ecthyma gangrenosum is an uncommon manifestation, usually secondary to cutaneous infection from either hematogenous seeding of a pathogen or direct inoculation through the skin. ${ }^{[2]}$ It usually occurs in patients who are critically ill and immunocompromised or neutropenic. ${ }^{[3]}$ Although ecthyma gangrenosum is now known to be caused by a variety of bacteria and fungi, once it was considered to be pathognomonic for $P$. aeruginosa infection and it still continues to be the most common cause ${ }^{[4]}$ However, ecthyma gangrenosum occurs in only in less than $6 \%$ of patients with Pseudomonas sepsis. ${ }^{[5]}$

The skin lesions usually occur in the gluteal and perineal regions $(57 \%)$ or extremities $(30 \%){ }^{[6]}$ Bacterial invasion of the arteries in the dermis and subcutaneous tissues producing a necrotizing vasculitis occurs by hematogenous seeding in bacteremic patients. The pathogenic mechanism, a vasculitis, is caused by enzymes and toxins liberated by the bacteria multiplying in the subcutaneous tissue. ${ }^{[7]}$ Protease and endotoxin A produced by $P$. aeruginosa are responsible for the direct tissue destruction and ulcerative lesions. In addition, elastases produced by the bacilli destroy the elastic lamina of small vessels, leading to hemorrhage and release of organisms into the surrounding tissue. ${ }^{[7]}$ The characteristic clinical appearance of ecthyma gangrenosum is a red macule that progresses to a nodular or ulcerative lesion with central area of necrosis surrounded by erythema. This lesion usually represents a potentially life-threatening systemic infection. The pathogen can be cultured from the skin lesion. Histologic findings show a necrotizing vasculitis with minimal inflammation and extensive tissue necrosis. Mortality rates of patients with ecthyma gangrenosum vary between $35 \%$ and $90 \%$ depending on the severity of the underlying sepsis. ${ }^{[5]}$ In patients without documented bacteremia, mortality rates can be as low as $15 \%{ }^{[8]}$

This patient had catheter-related pseudomonas infection with secondary seedling to the limb leading to "ecthyma gangrenosum of a single limb" in which the arterial line was placed. Early recognition, removal of the catheter, and systemic treatment of the underlying sepsis lead to the survival of this patient.

\section{References}

1. Funk E, Ivan D, Gillenwater AM. Ecthyma gangrenosum: an unusual cutaneous manifestation of the head and neck. Arch Otolaryngol Head Neck Surg 2009;135:818-20.

2. Zomorrodi A, Wald ER. Ecthyma gangrenosum: considerations in a previously healthy child. Pediatr Infect Dis J 2002;21:1161-4.

3. Musher DM. Cutaneous and soft-tissue manifestations of sepsis due to gram-negative enteric bacilli. Rev Infect Dis 1980;2:854-64.

4. Bodey GP, Bolivar R, Fainstein V, Jadeja L. Infections caused by Pseudomonas aeruginosa. Rev Infect Dis 1983;5:279-313.

5. Huminer D, Siegman-Igra Y, Morduchowicz G, Pitlik SD. Ecthyma gangrenosum without bacteremia: Report of six cases and review of the literature. Arch Intern Med 1987;147:299-301.

6. Solowski NL, Yao FB, Agarwal A, Nagorsky M. Ecthyma gangrenosum: a rare cutaneous manifestation of a potentially fatal disease. Ann Otol Rhinol Laryngol 2004;113:462-4.

7. Sevinsky LD, Viecens C, Ballesteros DO, Stengel F. Ecthyma gangrenosum: A cutaneous manifestation of Pseudomonas aeruginosa sepsis. J Am Acad Derm 1993;29:104-6.

8. Bodey GP, Jadeja L, Elting L. Pseudomonas bacteremia: Retrospective analysis of 410 episodes. Arch Intern Med 1985;145:1621-9.

How to cite this article: Varghese GM, Eapen P, Abraham S. Ecthyma gangrenosum of a single limb. Indian J Crit Care Med 2011;15:188-9.

Source of Support: Nil, Conflict of Interest: None declared. 